\title{
Does incongruence of lexicosemantic and prosodic information cause discernible cognitive conflict?
}

\author{
RACHEL L. C. MITCHELL \\ University of Reading, Reading, England \\ and Institute of Psychiatry, London, England
}

\begin{abstract}
We are often required to interpret discordant emotional signals. Whereas equivalent cognitive paradigms cause noticeable conflict via their behavioral and psychophysiological effects, the same may not necessarily be true for discordant emotions. Skin conductance responses (SCRs) and heart rates (HRs) were measured during a classic Stroop task and one in which the emotions conveyed by lexicosemantic content and prosody were congruent or incongruent. The participants' task was to identify the emotion conveyed by lexicosemantic content or prosody. No relationship was observed between HR and congruence. SCR was higher during incongruent than during congruent conditions of the experimental task (as well as in the classic Stroop task), but no difference in SCR was observed in a comparison between congruence effects during lexicosemantic emotion identification and those during prosodic emotion identification. It is concluded that incongruence between lexicosemantic and prosodic emotion does cause notable cognitive conflict. Functional neuroanatomic implications are discussed.
\end{abstract}

\section{Conflicting Emotional Signals}

Everyday communications constantly require us to decipher emotional signals, to interact with other people in a successful and appropriate manner (Trauner, Ballantyne, Chase, \& Tallal, 1993), and to maintain social relationships (Carton, Kessler, \& Pape, 1999). To do this, we rely on various cues, including facial emotion, gestures, (lexico-)semantic content, and prosody. Communication and interpretation of emotion is a multidetermined behavior (Borod, 1993). However, we are repeatedly confronted with situations in which sources of emotional information contradict one other. Someone may describe a situation using words leading listeners to believe he or she is happy, but his or her facial expression may convey a different emotion; or a person may describe the same situation using an angry tone of voice. In these scenarios, the protagonist may wish to hide his or her true opinions or feelings. It is only in cases such as irony, banter, and sarcasm that the incongruence itself possesses a particular, interpretable meaning.

\section{Cognitive Conflict}

In cognitive psychology, the Stroop task has become a classic paradigm with which to study the correlates of conflict and factors that modulate it. In the classic task, participants are required to identify the ink color in which

This study was conducted while the author was a lecturer in the Department of Psychology at Keele University, Staffordshire, England. Correspondence concerning this article should be addressed to R. L. C. Mitchell, School of Psychology, University of Reading, Whiteknights Road, Reading, Berkshire RG6 6AL, England (e-mail: r.l.c.mitchell@rdg.ac.uk). color words are printed. However, they find it difficult to suppress the more automatic process of reading. The occurrence of conflict at the cognitive level can be inferred from various measures, including certain patterns of behavioral and psychophysiological responses. At the behavioral level, participants are typically faster at naming the ink color for stimuli in which ink color and color word are congruent than for stimuli in which they are incongruent (MacLeod, 1991; Stroop, 1935). Skin conductance responses (SCRs) are the rapid, transient changes in conductance on the surface of the skin that accompany changes in the autonomic innervation of eccrine sweat glands via the sympathetic nervous system. Increased cognitive and behavioral demands often lead to increased autonomic nervous system activity, with consequent increases in sweat gland activity, which are particularly noticeable in the fingers and palms of the hands due to their high concentration of sweat glands. When participants are processing incongruent stimuli in the Stroop task, their behavioral reactions are usually accompanied by a noticeable increase in SCR (Tulen, Moleman, van Steenis, $\&$ Boomsma, 1989), indicating increased stress and arousal at the peripheral nervous system level (Hugdahl, 1995).

\section{Detecting SCR Responses to Emotional Stimuli}

When processing the emotional signals they encounter, individuals display affective, behavioral, and facial reactions, as well as visceral reactions such as fluctuations in SCR (Lang, Greenwald, Bradley, \& Hamm, 1993). A multifaceted response to emotions is intuitive given the evidence for associations between these different systems. Indeed, James (1884) claimed that emotions are the autonomic nervous system's response to emotion-provoking stimuli. Furthermore, direct neural pathways exist between 
long-established emotional processing regions such as the amygdala and executive function regions such as the prefrontal cortex and the anterior cingulate cortex (ACC), and these pathways are thought to enable goal-directed behavioral responses to cognitive tasks (Holland \& Gallagher, 2004). These brain regions are among those known to influence SCR (Critchley, 2002). Thus, SCRs have now been found to be affected by facial, pictorial, videographic, and written emotional stimuli (Campos, Marcos, \& González, 1999; Gomez, Stahel, \& Danuser, 2004; Hubert \& de Jong-Meyer, 1990; Lang et al., 1993; Meadows \& Kaplan, 1994; Witvliet \& Vrana, 1995). Despite the interest in interactions between emotions and psychophysiological responses, comparatively little is currently known about the psychophysiological response to auditory emotion.

\section{Why Might Incongruent Emotional Information Not Cause Cognitive Conflict?}

The key element of paradigms typically used to induce cognitive conflict (e.g., the Stroop task) is the interdimensional conflict of task stimuli. Since this feature is present in speech in which lexicosemantic and prosodic cues convey different emotions, a cursory consideration of this similarity might cause one to think that emotional incongruence in speech would similarly induce cognitive conflict. However, there are reasons to suspect that this might not necessarily be the case. In children, the ability to decode emotional signals develops at a surprisingly early age, with infants as young as 3 years old achieving nearly perfect performance on tests of facial and prosodic emotion recognition (Brosgole \& Weisman, 1995). Often, when a task or ability is practiced repeatedly, a person becomes quite proficient, sometimes to the point of internalizing the ability as a skill and reducing the conscious effort needed to perform the ability again (Shanks \& Johnstone, 1999). Interpersonal skills can similarly be improved by practice (McConnell, 2004). Thus, if we learn the ability to decode emotional signals at an early age and continue to engage in social interactions requiring these skills, by adulthood a healthy individual should be highly skilled at performing this ability. As for the added demands posed by incongruent emotional signals, evidence from classic Stroop task paradigms (MacLeod \& Dunbar, 1988; Melara, Rao, \& Tong, 2002) would suggest that the cognitive conflict posed by incongruous task-relevant and -irrelevant dimensions similarly diminishes with practice, although both the cognitive and the emotional forms may require extended periods of practice.

\section{Previous Applications of Related Tasks}

Although paradigms incorporating conflict between lexicosemantic and prosodic emotion cues have been used in previous research (see Table 1), behavioral evidence of between-dimensions congruence effects is inconsistent (see below). For this reason alone, dependence on behavioral performance measures may not provide a clear or reliable answer to the question of whether or not lexicosemantic/prosodic incongruence causes cognitive conflict. Furthermore, whereas we may mask or alter our true behavioral reactions via conscious choice, it is much more difficult to consciously choose to alter our psychophysiological reactions (Dollins, Cestaro, \& Pettit, 1998). These may therefore represent a more objective index of the response to cognitive conflict. Event-related potentials (ERPs) have been used to index the central nervous system's response to incongruence between lexicosemantic and prosodic cues. In such a study, Schirmer and Kotz (2003) interpreted an exaggerated N400 response as evidence of a Stroop-type effect. However, even in healthy populations ERP responses are subject to broad interparticipant variability of peak latencies and amplitudes, the specificity of some ERP tests is questionable (Barrett, 2000), and others have raised doubts about the separability of the endogenous and exogenous components of the ERP response (Gaillard, 1988). Another means of assessing the internal response to lexicosemantic/prosodic incongruence may therefore be prudent. In the present study, discord between the emotions conveyed by lexicosemantic and prosodic cues was examined as it would occur in most everyday speech - that is, in sentences. Previous applications of these paradigms have been used to examine the effects of congruence in relation to isolated words (Grimshaw, 1998; Kitayama \& Ishii, 2002; Schirmer \& Kotz, 2003) and carrier sentences in which the lexicosemantic and prosodic information of interest is limited to the last word (Wurm \& Vakoch, 1996; Wurm, Vakoch, Strasser, Calin-Jageman, \& Ross, 2001).

\section{Hypotheses and Rationales}

In the study outlined below, the following hypotheses were tested.

Hypothesis 1. SCR is greater when incongruent lexicosemantic and prosodic emotions are processed than when congruent emotions are processed. A discussion of this hypothesis is given above. At the behavioral level, data (from paradigms in which lexicosemantic and prosodic information are varied in congruence) appear equivocal, comprising both reports of supporting evidence (Grimshaw, 1998; Schirmer \& Kotz, 2003) and reports of contradictory evidence (Wurm \& Vakoch, 1996; Wurm et al., 2001).

Hypothesis 2. The effect of congruence on SCR during response to lexicosemantic emotion is different from that during response to prosodic emotion. At the behavioral level, the evidence is again contradictory. According to Kitayama and Ishii (2002), incongruent lexicosemantics and prosody caused cognitive conflict only when participants made judgments about prosody. Grimshaw (1998) also found that the effect of congruence was larger for judgments about prosody than for judgments about lexicosemantic emotion, but the effect of congruence for lexicosemantic judgments was still significant. In contrast, Schirmer and Kotz (2003) found that the congruence effect was stronger for lexicosemantic judgments than for judgments about prosody.

Hypothesis 3. The difference between the rise in SCR (from baseline) caused by incongruence and that caused by congruence of information from task-relevant and-irrelevant 
Table 1

Studies Implementing Paradigms That Incorporate Conflict Between Lexicosemantic and Prosodic Emotion Cues

\begin{tabular}{ll}
\hline \multicolumn{1}{c}{ Study } & \multicolumn{1}{c}{ Purpose } \\
\hline Grimshaw (1998) & To examine the interaction between \\
& lexicosemantic/prosodic informa- \\
& tion and hemispheric specializa- \\
tion style (complementary [two & dimensions processed in different \\
& hemispheres] vs. noncomplemen- \\
& tary [both dimensions processed in \\
& same hemisphere] specialization).
\end{tabular}

Dichotic listening task. The words $\mathrm{mad}$, sad, glad, and fad spoken in angry, sad, happy, and neutral tones of voice. Participants attended to lexicosemantic content or tone of voice and indicated whether target information was present or absent in either ear.

Positive and negative words spoken in pleasant and harsh tones of voice. Participants instructed to attend to one dimension while ignoring the other. Both dimensions tested in separate blocks. Self-paced task. but primarily to prosodic emotion in a high-context culture/language (Japanese).

Schirmer \& Kotz (2003) To compare interaction of emotional prosody and lexicosemantic valence during emotional comprehension in men and women.

Wurm \& Vakoch (1996)

To explain affective lexicon in terms of three underlying dimensions: evaluation, activity, and potency; to assess importance of these dimensions during speech perception.

Wurm et al. (2001)

To determine whether or not prosodic expression of emotion affected speed with which listeners could identify emotion words.
ERP study. Positive, negative, and neutral verbs spoken with happy, neutral, and angry prosody. Participants instructed to attend to one dimension while ignoring the other. Both dimensions tested in separate blocks. ISI, 3-3.2 sec.

Pure emotions (disgusted, petrified, and happy) and equivalent nonwords* inserted at end of a single carrier phrase. Phrase read in happy, petrified, disgusted, and neutral tones of voice. Participants made speeded lexical decision about target word.

See Wurm and Vakoch (1996).
Main Findings

Lexicosemantic information interfered more with prosodic processing than prosodic information did with lexicosemantic processing, although prosodic information did produce significant interference. Failed to support the hypothesis that corpus callosum shields each hemisphere from the other to permit independent, parallel processing - complementarity had no consequences for the integration or interference of lexicosemantic and prosodic information.

Interference effect of competing lexicosemantic evaluation in prosodic emotion judgment was stronger in English. Interference effect of competing prosodic emotion in lexicosemantic judgment stronger in Japanese.

Comparable behavioral effects between men and women: reactions to congruent stimuli faster and more accurate (and more salient for the lexicosemantic condition). ERP showed smaller N400 amplitude for congruent stimuli, but significant only for lexicosemantic judgment and female listeners.

Tone of voice did not influence decision times or interact with lexicosemantic category. Lexical decision times significantly predicted by interaction between evaluation, activity, and potency dimension weights.

Randomized presentation of tone of voice showed no effect of congruence. Blocked presentation of tone of voice did show effect of congruence.

Note-ISI, interstimulus interval. *Each equivalent nonword was derived by changing the phoneme of the corresponding word at the point in the acoustic signal where it diverges from all other English words.

stimulus dimensions is greater for the classic Stroop task than it is for the auditory emotional interference task. For the reasons posed above regarding practice at this social skill, it is hypothesized that if emotional incongruence does induce cognitive conflict, the effect will be notably smaller than that for classic inducers of cognitive conflict.

\section{METHOD}

The protocol of this study was approved by the ethics committee of the Department of Psychology of Keele University, Staffordshire, England.

\section{Participants}

Forty-four psychiatrically normal participants (36 females and 8 males) at a mean age of 18.7 years $( \pm 1.3)$ were recruited from among undergraduates in the Department of Psychology of Keele University and A-level students from local sixth form colleges.
Absence of psychiatric illness was established via self-report. To avoid confounding behavioral data, volunteers with color blindness or hearing problems were excluded. To facilitate accurate psychophysiological measurements, volunteers with high blood pressure or heart problems were excluded.

\section{Experimental Paradigms}

In this study, the effects of interdimensional incongruence in the auditory emotional interference paradigm were compared with those in a classic color Stroop task. Inclusion of the classic color Stroop task served two main purposes. First, it functioned as a control task to rule out the possibility that negative results for the auditory emotional interference paradigm may be caused by faulty equipment or procedure; second, it functioned as a standard comparator for gauging the size of any cognitive conflict induced by the auditory emotional interference paradigm. The order of the two tasks was counterbalanced across participants.

Classic color Stroop task. Two series of 40 color words (randomly selected from red, green, blue, and brown) were presented in 
the center of the screen (every $2 \mathrm{sec}$, at 0.5 -sec intervals), in different color fonts (red, green, blue, and brown) and in 18-point Arial typeface. For the congruent condition, color words were displayed (in a single block) in matching font colors; in the incongruent condition, they were displayed (again in a single block) in a conflicting color (e.g., the word RED was displayed in blue). In each condition, the participants were instructed to indicate the font color as quickly as they could by pressing the " 1 " key for red, the " 2 " key for green, the " 3 " key for blue, or the " 4 " key for brown. The order of the congruent and incongruent blocks was alternated between participants.

Auditory emotional interference task. Prerecorded sentences were used for which the emotion (happy or sad) conveyed by lexicosemantic content (e.g., "The dog had to be put down," "She won the lottery jackpot") either matched that conveyed by prosody, as in the two congruent conditions, or conflicted with it, as in the two incongruent conditions. Sentences were approximately the same length and consistent in style and format. A survey $(n=20)$ determined perceived happiness and sadness, and those sentences rated closest to the happy and sad ends of the scale were recorded by an experienced male phonetician in happy and sad emotional intonations. Audiocassette recordings were digitized at $22 \mathrm{kHz} / 16$ bits. Additional background details on the generation of these stimuli can be found in Mitchell, Elliott, Barry, Cruttenden, and Woodruff (2003). Stimuli in which relevant lexicosemantic information was presented across the entire sentence (as opposed to in an isolated word in a carrier sentence) were given preference to avoid interpretation difficulties caused by differences in stimulus onset asynchronies between lexicosemantic and prosodic elements (Wurm \& Vakoch, 1996).

In each condition, 20 sentences were randomly selected from the prerecorded set, with happy and sad scenario sentences being equal in number and randomly interspersed. Within each condition, 1 sentence was played every $5 \mathrm{sec}$, in a single block, via backgroundnoise-attenuating headphones. In the congruent and incongruent attend-semantic-content conditions, the participants' task was to concentrate on the emotion conveyed by the semantic content and to indicate whether they heard a sentence about a happy scenario or one about a sad scenario by pressing the " $\mathrm{H}$ " key (using the dominant hand) for happy sentences or the "S" key for sad sentences. In the prosody conditions, the participants' task was to concentrate on the emotion conveyed by tone of voice and to indicate whether the speaker used a happy tone or a sad tone of voice (again using the " $\mathrm{H}$ " and "S" keys, respectively). The participants were instructed not to wait until the end of a sentence to respond; they could indicate their responses as soon as they had made their judgments. The order of the four blocks was fully counterbalanced across participants.

The color and auditory emotional interference tasks shared the following features: (1) Both tasks were block design paradigms. (2) Mean phasic SCR responses to stimuli were collected for both tasks. (3) Both tasks were presented via a standard PC. (4) Both tasks required a manual keyboard response via a single key. (5) Both tasks incorporated interference at the response selection/preparation stage (i.e., the irrelevant stimulus attribute clashed with the required response because the response preparation and interference processes shared the verbal modality). (6) The conditions within both tasks were counterbalanced to avoid priming effects. (7) The duration of the conditions was the same in both tasks $(100 \mathrm{sec})$. (8) In both tasks, the task-relevant and task-irrelevant dimensions were part of the same percept (unlike in the counting Stroop task, for example; Bush et al., 1998).

\section{Acquisition of Psychophysiological Data}

Psychophysiological data were acquired in a room controlled for noise and temperature (mean temperature $=22.2^{\circ} \mathrm{C} \pm 1.78^{\circ} \mathrm{C}$ ). Prior to data collection, the participants' hands were washed with mild soap and water. Each participant's test session began with a 10-min rest period during which his or her heart rate (HR) and SCR acclimated to the environment. A 3-min rest period followed each condi- tion to allow the participant's HR and SCR to settle toward baseline. $\mathrm{HR}$ and SCR were recorded continuously using a BIOPAC MP-100 remote monitoring system (BIOPAC Systems, Goleta, CA; www .biopac.com). HR was obtained (in beats per minute) by detecting the $\mathrm{R}$ wave from EKG electrodes placed on each wrist, with a reference electrode on the nondominant forearm. SCR was measured (in $\mu$ mhos) using 8-mm standard silver/silver chloride electrodes filled with an isotonic conductive jelly $(0.05$ molar $\mathrm{NaCl})$ and attached to the distal phalanx of the index and middle fingers of the nondominant hand. Importantly, doing so left the dominant hand free for behavioral responses. The participants were instructed to rest their hands immediately in front of the keyboard between stimuli, to minimize the movement and time required to make responses. A constant voltage of $0.5 \mathrm{~V}$ was applied across the electrodes, and the lower frequency response was set to DC to obtain absolute skin conductance levels. Output from all electrodes was recorded on a standard PC using BIOPAC Systems AcqKnowledge software. During acquisition of the psychophysiological data, the participants were asked to remain as still as possible to avoid confounding these measurements.

\section{Analyses}

Behavioral data. For both tasks, the dependent behavioral measures were mean performance accuracy and mean reaction time (RT) to correct responses. For the control task, data from each condition were entered into a paired-samples $t$ test analysis to determine whether or not RTs differed significantly for the incongruent and congruent conditions. For the auditory emotional interference task, data were entered into a 2 (emotion dimension: lexicosemantic content vs. prosody) $\times 2$ (congruence: congruent vs. incongruent) within-subjects ANOVA. To compare the extent of congruence effects between tasks, mean RT from the congruent condition was subtracted from that in the incongruent condition, to create a single congruence-effect-size measure for each task (collapsed across the lexicosemantic and prosodic conditions for the experimental task). Scores for the two tasks were then compared directly in paired $t$ tests. Parallel between-tests comparisons of performance accuracy data were not possible due to ceiling effects on performance accuracy in the classic Stroop task.

Psychophysiological data. For both tasks, phasic SCRs were isolated using AcqKnowledge to determine mean skin conductance levels during stimulus presentation, excluding that during interstimulus intervals. For the analyses described, mean HR responses were similarly calculated over the period of stimulus presentation, excluding interstimulus intervals. All SCR and HR data were corrected for baseline levels of responsiveness; the mean measurement for the baseline immediately preceding each condition was subtracted from the mean measurement for each condition. If significant or repeated artifacts were observed in the traces during any condition, the corresponding data set was classified as missing data. Minor or isolated artifacts were corrected by removing the affected part of the trace and connecting the disjointed end points.

Control task. For the classic Stroop task, SCR and HR data were entered into separate paired-sample $t$ test analyses to determine whether or not measurements for the incongruent condition differed significantly from those for the congruent condition.

Auditory emotional interference task. For the experimental task, SCR and HR data for each condition were entered into separate within-subjects factorial ANOVAs comprising two factors: emotion dimension (lexicosemantic content vs. prosody) and congruence (congruent vs. incongruent).

Between-tasks differences. To compare the extent of congruence effects between the tasks, baseline corrected SCR and HR in the congruent condition were subtracted from SCR and HR in the incongruent condition, to create a single congruence-effect-size measure for each task (collapsed across the lexicosemantic and prosodic conditions for the experimental task). The scores for the two tasks were then compared directly in a paired $t$ test. 


\section{RESULTS 1}

The behavioral and psychophysiological data are presented in Table 2.

\section{Behavioral Data}

Classic Stroop task. The participants took significantly longer to name the font color in the incongruent condition than in the congruent condition $[t(43)=21.54$, $p<.001]$ and were also less accurate in the incongruent than in the congruent condition $[t(43)=2.76, p<.01]$.

Auditory emotional interference task. There was no effect of the emotion dimension $[F(1,43)=0.30, p=.59]$ on RT; RTs were similar whether the participants were responding to the emotion conveyed by lexicosemantic content or to that conveyed by prosody. There was, however, a significant effect of congruence $[F(1,43)=5.19$, $p<.05]$ : The participants took longer to respond to stimuli in which the emotions conveyed by lexicosemantic content and by prosody conflicted. The interaction between emotion dimension and congruence was not significant $[F(1,43)=0.90, p=.35]$ : The effect of congruence on the participants' RTs was similar whether they were responding to lexicosemantic or to prosodic emotion. The pattern of results was similar for performance accuracy: The participants performed as accurately when attending to the emotion conveyed by lexicosemantic content as when attending to that conveyed by prosody $[F(1,43)=$ $0.03, p=.88]$ and less accurately in the incongruent than in the congruent condition $[F(1,43)=8.15, p<.01]$. The interaction between emotion dimension and congruence was not significant $[F(1,43)=3.369, p=.073]$.

Between-tests comparisons of the effect of congruence on RT revealed that between-dimensions incongruence in the classic Stroop task induced significantly greater RT increases (over RTs to congruent stimuli) than were observed for the auditory emotional interference task $[t(43)=7.64, p<.01]$.

\section{Psychophysiological Data}

Classic Stroop task. The participants' SCRs were significantly higher in the incongruent than in the congruent condition $[t(43)=2.53, p<.05]$. In contrast, there was no difference between the participants' HRs in the congruent and incongruent conditions $[t(43)=1.56, p=$ .125].

Auditory emotional interference task. Preliminary comparisons of SCR and HR during the preceding baseline established that baseline SCR and HR measures did not differ significantly from one condition to another $[F(1,43)=2.10, p=.12$ for SCR; $F(1,43)=0.76, p=$ .42 for HR], thus avoiding the confounds suggested by Wilder's (1967) law of initial values. For the HR data analyses, the main effects of emotion dimension and congruence were not significant $[F(1,43)=0.40, p=.53$ and $F(1,43)=0.45, p=.51$, respectively]. The interaction between emotion and congruence was also not significant $[F(3,129)=0.90, p=.35]$. For the SCR data, there was no main effect of emotion dimension $[F(1,43)=1.94$, $p=.17]$. The main effect of congruence was significant in that it was significantly higher in the incongruent than in the congruent condition $[F(1,43)=4.11, p<.05]$. The interaction between emotion and congruence was not significant $[F(3,129)=2.94, p=.09]$.

Between-tasks differences. For the SCR data, congruence effect scores were significantly higher for the classic Stroop task than for the auditory emotional interference $\operatorname{task}[t(43)=2.82, p<.01]$, but for the HR data the difference between the congruence effect scores for the two different tasks was not significant $[t(43)=1.23, p=.23]$.

\section{DISCUSSION}

\section{Effect of Congruence}

As was the case with the classic Stroop task, interdimensional incongruence in the auditory emotional interference task caused a significant increase in SCR. Furthermore, this increase was similar whether participants were identifying the emotion conveyed by lexicosemantic content or that conveyed by prosody. The SCR pattern of response was paralleled by behavioral responses in that participants were slower to respond in the incongruent condition than in the congruent condition. These results suggest that, whether participants attend to lexicosemantic or to prosodic emotion, the task-irrelevant dimension causes interference with the task-relevant dimension that is difficult to inhibit. Since SCR is believed to be related to arousal, the increased cognitive demand of the incon-

Table 2

Summary Descriptive Statistics

\begin{tabular}{|c|c|c|c|c|c|c|c|c|c|}
\hline \multirow[b]{2}{*}{ Task } & \multirow[b]{2}{*}{ Condition } & \multicolumn{2}{|c|}{ Accuracy (\%) } & \multicolumn{2}{|c|}{$\mathrm{RT}(\mathrm{sec})$} & \multicolumn{2}{|c|}{$\mathrm{HR}^{*}$ (bpm) } & \multicolumn{2}{|c|}{$\mathrm{SCR}^{*}(\mu \mathrm{mho})$} \\
\hline & & $M$ & $S D$ & $M$ & $S D$ & $M$ & $S D$ & $M$ & $S D$ \\
\hline \multirow[t]{2}{*}{ Color Stroop } & Congruent & 99.98 & 0.13 & 0.68 & 0.08 & 7.43 & 0.93 & 1.18 & 0.12 \\
\hline & Incongruent & 98.36 & 3.90 & 1.28 & 0.17 & 9.30 & 1.12 & 1.86 & 0.26 \\
\hline \multicolumn{10}{|l|}{ Experimental } \\
\hline \multirow[t]{2}{*}{ Attend semantics } & Congruent & 91.48 & 8.11 & 2.38 & 0.26 & 0.52 & 4.01 & 0.75 & 0.72 \\
\hline & Incongruent & 89.55 & 14.66 & 2.72 & 0.18 & -0.65 & 3.00 & 0.79 & 0.77 \\
\hline Attena prosody & Con & 94.55 & 6.36 & 2.52 & 0.2 & -0.65 & 3.69 & 0.55 & 0.57 \\
\hline & Incongruent & 87.05 & 14.72 & 2.81 & 0.56 & 0.98 & 12.41 & 0.87 & 0.69 \\
\hline
\end{tabular}

Note- RT, reaction time; HR, heart rate; SCR, skin conductance rate. ${ }^{*}$ Following correction of the data for baseline values. 
gruent condition appears to have the psychophysiological consequence of increasing arousal-perhaps much as the incongruent condition of the classic Stroop task acts as a cognitive stressor-but, in a format with greater ecological validity. According to Selye (1956), stress results when situational demands are perceived to exceed available coping resources, exemplified here by the presumably increased demand of the incongruent condition and its concurrent detrimental effects on performance.

Although the interpretation of these results seems straightforward, the lack of previous consistency is somewhat puzzling. Some studies report a lack of congruence effects between lexicosemantic emotion and prosodic emotion (Wurm \& Vakoch, 1996; Wurm et al., 2001). However, these studies incorporated a task quite unlike those of most other studies of this ilk-namely, a lexical decision task about whether emotion items at the end of carrier sentences were real words or nonwords. With these stimuli, prosodic information starts from the beginning of the sentence whereas emotional lexicosemantic information does not begin until near the end. Such a task also ignores any reverse influence of lexicosemantic emotion on prosodic emotion interpretation. The interdimensional interference in these tasks may not be strong enough to generate cognitive conflict. Bradley and Lang (2000) suggested that, since new sensory information is added serially during presentation of auditory material spanning several seconds, physiological systems that respond to simple changes in the stimuli may be continuously active, making emotional signals difficult to detect. Nevertheless, like the shorter word-length stimuli presented by Grimshaw (1998) and Schirmer and Kotz (2003), it was still possible in the present study for participants to detect emotional signals and to respond behaviorally and physiologically to the incongruence. As Schirmer and Kotz themselves suggested, using both semantically neutral and prosodically neutral stimuli may be an additional way to increase task demands to such a degree that congruence effects become more prevalent, although doing so could mean that participants no longer seek to identify the emotion conveyed by the relevant dimension; by periodically comparing neutral information to emotional information, they may in fact be thinking about the signal's intensity, a factor believed to relate to psychophysiological responses themselves (Lang, Bradley, \& Cuthbert, 1998).

Regarding whether or not prosodic information interferes with the processing of lexicosemantic information more than vice versa, previous reports are perhaps more contradictory. In the present study, there was a trend toward interaction between emotion and congruence, the effect of congruence on SCR appearing to be larger for prosody than for semantics. However, this difference did not reach statistical significance, and neither did that from the corresponding behavioral data. Kitayama and Ishii (2002) proposed that because English is a "low-context" language, English speakers rely less on contextual clues (such as prosody) and more on lexicosemantic content. They found some support for the idea that the greatest interference experienced by English speakers was that of contrasting lexicosemantic evaluation on prosody evaluation. One could argue that the use of a verbal response label predisposes participants to lexicosemantic processing even when the task requires them to focus on prosodic information. In contrast, Wurm et al. (2001) argued for the importance of contextual information such as emotional prosody, since it appears to constrain our interpretation not just of emotional words but of nonemotional ones too. Schirmer and Kotz's (2003) findings support those of Wurm et al., but at the central nervous system level, their ERP data implied that this pattern of response was limited to female participants. Although the imbalanced gender ratio could be considered a limitation of the present study, removal of the male participants from the data set did not alter the significance patterns obtained at the behavioral or SCR level. In summary, currently available data provide evidence for the potential of significant cognitive conflict in both directions. Indeed, the present study provides behavioral indications of cognitive conflict regardless of which of the two dimensions is task relevant. However, it extends the results of previous literature by its original demonstrations of cognitive conflict during incongruent emotion via peripheral nervous system indicators. Despite our perpetual exposure to both sources of auditory emotion (and their episodic incongruence), and despite the consequent potential equivalence in practice and automaticity, we should perhaps not discard the possibility that cognitive conflict resulting from incongruence of lexicosemantic and prosodic emotion may be flexible (Grimshaw, 1998).

\section{Comparing the Tasks}

The mean increase in SCR from baseline for the incongruent condition of the classic Stroop task was $16.4 \%$, whereas that for the auditory emotional interference Stroop task was only $7.5 \%$. In addition, analysis of congruence effect sizes revealed that the difference in the SCR's increase between the incongruent and congruent conditions was much larger in the classic Stroop task than in the experimental task. Similarly significant increases were observed in the accompanying behavioral (RT) data. The obvious explanation of this difference might simply be that the interference effect associated with the classic Stroop task is larger than that associated with the experimental task. One possible explanation for the apparent difference in effect size is that of different complexities in the possible number of conflict exemplars. Whereas the auditory emotional interference task incorporated two conflict exemplars (happy sentences spoken in a sad tone of voice, and sad sentences spoken in a happy tone of voice), the classic color Stroop task incorporated several more (e.g., the word RED in blue font, the word RED in green font, the word BLUE in red font, and so on). However, the ratio of congruent to incongruent stimuli was the same in both tasks. One might also consider the duration of the stimuli in each task to be a potential explanatory factor. Whereas in the classic color Stroop task participants have to read a single word, in the auditory emotional interference paradigm their behavioral and SCR responses reflect a longer period of stimulation. However, 
the purpose of the auditory emotional interference paradigm was to represent, as closely as possible (while retaining appropriate experimental testing conditions), the social situations in which we encounter conflicting emotion cues. In these situations, we are more likely to encounter and have to process sentences than isolated words. Thus, to assess cognitive conflict in these situations as naturalistically as possible, it was necessary to assess responses to sentences rather than responses to isolated words.

The smaller psychophysiological effect of the auditory emotional interference task might also be related to its commonplace nature. Although it is unlikely that most people have to distinguish between font colors and color words in their daily lives, we are repeatedly faced with situations in which emotional communications are contradictory. In accordance with arguments raised in the introduction, it is possible that everyday practice in this skill makes the incongruent condition of the auditory emotional interference Stroop task less cognitively stressful for participants than the color task; hence the lower SCR in the former task. However, the statistical significance of the congruence factor in the auditory emotional interference Stroop task means that it is still an efficient source of cognitive conflict. It remains somewhat unclear, though, whether its effects represent the same type of cognitive conflict as that induced by classic cognitive tasks such as the Stroop test. Further neuroimaging studies may prove particularly beneficial in this respect (see Conclusions and Functional Neuroanatomic Implications, below). Similar neural mediators would suggest that these two sources of conflict represent a single process or mechanism.

\section{HR Responses}

In contrast to the clear relationship between SCR and congruence, little effect of congruence was observed on HR. This result is perhaps not surprising, considering the lack of predictable HR response to emotional stimuli of other modalities (Gendolla \& Krüsken, 2001; Gomez et al., 2004). Similarly, a reliable difference in HR between the incongruent condition and the neutral and congruent conditions is not always present in psychophysiological studies of the classic Stroop task (Silva \& Leite, 2000). It seems that SCR changes are poorly correlated with HR changes (Gendolla \& Krüsken, 2001). This observation may well reflect the difference in innervation of these two psychophysiological indices: innervation of the eccrine sweat glands that contribute to SCR by the sympathetic division of the autonomic nervous system, and antagonistic innervation of the cardiovascular system by the sympathetic and parasympathetic divisions. Indeed, emerging evidence suggests that different components of the autonomic nervous system may have different neural concomitants in the brain (Wittling, Block, Genzel, \& Schweiger, 1998; Wittling, Block, Schweiger, \& Genzel, 1998).

\section{Conclusions and Functional Neuroanatomic Implications}

Although its effects may be subtler than those present in standard cognitive paradigms, incongruence between dimensions of auditory emotion does cause notable cognitive conflict. Modulations of the effects of such emotional incongruence can be assessed from standard behavioral measures and certain psychophysiological measures. Early reports from neuroimaging studies suggest some similarities between mediation of performance on typical visual emotional Stroop tasks and that of performance on classic color Stroop tasks (Compton et al., 2003) in the brain, particularly in the dorsolateral prefrontal cortex. However, the lack of interdimensional conflict in visual emotional Stroop tasks does not make them an ideal choice for studying the effects of emotional incongruence in the brain. In their fMRI investigation, Schirmer, Zysset, Kotz, and von Cramon (2004) conceptualized semantic-prosodic incongruence as a "semantic anomaly." Their discussion highlighted the association between the activity of the inferior frontal gyrus - a region well-known for its semantic functions (see, e.g., P. M. Matthews et al., 2003) - and the resolution of this incongruence. However, the present study's multilevel demonstration of consequential cognitive conflict raises the possibility that these early studies may be missing a potentially important focal point. Various cognitive paradigms have shown a strong link between mediation of cognitive conflict and recruitment of the ACC (Botvinick, Cohen, \& Carter, 2004). On the basis of evidence from the present study, future neuroimaging studies of emotional incongruence should perhaps consider this brain region in their a priori hypotheses. Bearing in mind that the effects of cognitive conflict can be expressed through alterations in autonomic nervous system activity, evidence of functional links between ACC activity and autonomic control (Critchley, 2002; Critchley et al., 2003; S. C. Matthews, Paulus, Simmons, Nelesen, \& Dimsdale, 2004) add weight to this suggestion. Whereas classic cognitive Stroop tasks tend to activate the posterior portion of the ACC, an emotion-based version such as this one may be more likely to activate the anterior portion of the ACC (see Bush, Luu, \& Posner, 2000).

\section{REFERENCES}

Barrett, G. (2000). Clinical application of event-related potentials in dementing illness: Issues and problems. International Journal of Psychophysiology, 37, 49-53.

BoROD, J. C. (1993). Cerebral mechanisms underlying facial, prosodic, and lexical emotional expression: A review of neuropsychological studies and methodological issues. Neuropsychology, 7, 445-463.

Botvinick, M. M., Cohen, J. D., \& Carter, C. S. (2004). Conflict monitoring and anterior cingulate cortex: An update. Trends in $\mathrm{Cog}$ nitive Sciences, 8, 539-546.

BRADLEY, M. M., \& LANG, P. J. (2000). Affective reactions to acoustic stimuli. Psychophysiology, 37, 204-215.

Brosgole, L., \& Weisman, J. (1995). Mood recognition across the ages. International Journal of Neuroscience, 82, 169-189.

Bush, G., LuU, P., \& Posner, M. I. (2000). Cognitive and emotional influences in anterior cingulate cortex. Trends in Cognitive Sciences, 4, 215-222.

Bush, G., Whalen, P. J., Rosen, B. R., Jenike, M. A., McInerney, S. C., \& RAUCH, S. L. (1998). The counting Stroop: An interference task specialized for functional neuroimaging — validation study with functional MRI. Human Brain Mapping, 6, 270-282.

Campos, A., Marcos, J. L., \& González, M. A. (1999). Relationship between properties of words and elicitation of skin conductance response. Psychological Reports, 85, 1025-1030. 
Carton, J. S., Kessler, E. A., \& Pape, C. L. (1999). Nonverbal decoding skills and relationship well-being in adults. Journal of Nonverbal Behavior, 23, 91-100.

Compton, R. J., Banich, M. T., Mohanty, A., Milham, M. P., Herrington, J., Miller, G. A., ET Al. (2003). Paying attention to emotion: An fMRI investigation of cognitive and emotional Stroop tasks. Cognitive, Affective, \& Behavioral Neuroscience, 3, 81-96.

CRITCHLEY, H. D. (2002). Electrodermal responses: What happens in the brain? Neuroscientist, $8,132-142$.

Critchley, H. D., Mathias, C. J., Josephs, O., O'Doherty, J., Zanini, S., DewAR, B. K., ET AL. (2003). Human cingulate cortex and autonomic control: Converging neuroimaging and clinical evidence. Brain, 126, 2139-2152.

Dollins, A. B., Cestaro, V. L., \& Pettit, D. J. (1998). Efficacy of repeated psychophysiological detection of deception testing. Journal of Forensic Sciences, 43, 1016-1023.

GAILLARD, A. W. (1988). Problems and paradigms in ERP research. Biological Psychology, 26, 91-109.

GENDOLLA, G. H. E., \& KRÜSKEN, J. (2001). The joint impact of mood state and task difficulty on cardiovascular and electrodermal reactivity in active coping. Psychophysiology, 38, 548-556.

Gomez, P., StaheL, W. A., \& Danuser, B. (2004). Respiratory responses during affective picture viewing. Biological Psychology, 67, 359-373.

GRIMSHAW, G. M. (1998). Integration and interference in the cerebral hemispheres: Relations with hemispheric specialization. Brain \& Cognition, 36, 108-127.

Holland, P. C., \& GALlaGHer, M. (2004). Amygdala-frontal interactions and reward expectancy. Current Opinions in Neurobiology, 14 148-155.

Hubert, W., \& De Jong-Meyer, R. (1991). Psychophysiological response patterns to positive and negative film stimuli. Biological Psychology, 31, 73-93.

HUGDAHL, K. (1995). Psychophysiology: The mind-body perspective. Cambridge, MA: Harvard University Press.

JAMES, W. (1884). What is an emotion? Mind, 9, 188-205.

Kitayama, S., \& IshiI, K. (2002). Word and voice: Spontaneous attention to emotional utterances in two languages. Cognition \& Emotion, 16, 29-59.

LaNG, P. J., Bradley, M. M., \& Cuthbert, B. N. (1998). Emotion, motivation, and anxiety: Brain mechanisms and psychophysiology. Biological Psychiatry, 44, 1248-1263.

Lang, P. J., Greenwald, M. K., Bradley, M. M., \& Hamm, A. O. (1993). Looking at pictures: Affective, facial, visceral, and behavioral reactions. Psychophysiology, 30, 261-273.

MACLEOD, C. M. (1991). Half a century of research on the Stroop effect: An integrative review. Psychological Bulletin, 109, 163-203.

MACLEOD, C. M., \& DuNBAR, K. (1988). Training and Stroop-like interference: Evidence for a continuum of automaticity. Journal of Experimental Psychology: Learning, Memory, \& Cognition, 14, 126-135.

MATthews, P. M., AdCock, J., Chen, Y., Fu, S., Devlin, J. T., RuSHWORTH, M. F., ET AL. (2003). Towards understanding language organisation in the brain using fMRI. Human Brain Mapping, 18, 239-247.

Matthews, S. C., Paulus, M. P., Simmons, A. N., Nelesen, R. A., \& Dimsdale, J. E. (2004). Functional subdivisions within anterior cingulate cortex and their relationship to autonomic nervous system function. NeuroImage, 22, 1151-1156.

McConnel L, C. R. (2004). Interpersonal skills: What they are, how to improve them, and how to apply them. Health Care Management, 23, 177-187.

Meadows, M.-E., \& Kaplan, R. F. (1994). Dissociation of autonomic and subjective responses to emotional slides in right hemisphere damaged patients. Neuropsychologia, 32, 847-856.
Melara, R. D., Rao, A., \& Tong, Y. (2002). The duality of selection: Excitatory and inhibitory processes in auditory selective attention. Journal of Experimental Psychology: Human Perception \& Performance, 28, 279-306.

Mitchell, R. L. C., Elliott, R., Barry, M., Cruttenden, A., \& Woodruff, P. W. R. (2003). The neural response to emotional prosody, as revealed by functional magnetic resonance imaging. Neuropsychologia, 41, 1410-1421.

SchIRMER, A., \& KoTZ, S. A. (2003). ERP evidence for a sex-specific Stroop effect in emotional speech. Journal of Cognitive Neuroscience, 15, 1135-1148.

Schirmer, A., ZYsset, S., Kotz, S. A., \& von Cramon, D. Y. (2004). Gender differences in the activation of inferior frontal cortex during emotional speech perception. NeuroImage, 21, 1114-1123.

Selye, H. (1956). The stress of life. New York: McGraw-Hill.

Shanks, D. R., \& Johnstone, T. (1999). Evaluating the relationship between explicit and implicit knowledge in a sequential reaction time task. Journal of Experimental Psychology: Learning, Memory, \& Cognition, 25, 1435-1451.

SiLVA, F. T., \& LEITE, J. R. (2000). Physiological modifications and increase in state anxiety in volunteers submitted to the Stroop ColorWord Interference Test: A preliminary study. Physiology \& Behavior, 70, 113-118.

STROOP, J. R. (1935). Studies of interference in serial verbal reactions. Journal of Experimental Psychology, 18, 643-662.

Trauner, D. A., Ballantyne, A., Chase, C., \& Tallal, P. (1993). Comprehension and expression of affect in language-impaired children. Journal of Psycholinguistic Research, 22, 445-452.

Tulen, J. H. M., Moleman, P., van Steenis, H. G., \& Boomsma, F. (1989). Characterization of stress reactions to the Stroop Color Word Test. Pharmacology Biochemistry \& Behavior, 32, 9-15.

WILDER, J. (1967). Stimulus and response: The law of initial value. Bristol, U.K.: Wright.

Wittling, W., Block, A., Genzel, S., \& Schweiger, E. (1998). Hemisphere asymmetry in parasympathetic control of the heart. $\mathrm{Neu}$ ropsychologia, 36, 461-468.

Wittling, W., Block, A., Schweiger, E., \& Genzel, S. (1998). Hemisphere asymmetry in sympathetic control of the human myocardium. Brain \& Cognition, 38, 17-35.

WitvlieT, C. V. O., \& VRana, S. R. (1995). Psychophysiological responses as indices of affective dimensions. Psychophysiology, 32, 436-443.

WURM, L. H., \& VAKoch, D. A. (1996). Dimensions of speech perception: Semantic associations in the affective lexicon. Cognition \& Emotion, 10, 409-423.

WURm, L. H., VaKoCh, D. A., Strasser, M. R., Calin-Jageman, R., \& Ross, S. E. (2001). Speech perception and vocal expression of emotion. Cognition \& Emotion, 15, 831-852.

\section{NOTE}

1. Since women represented the majority gender in the participant sample, data from the female participants were analyzed separately to exclude possible partial confounds from data from the male participants. Analysis of the data of the female participants alone did not alter the significance patterns obtained at the behavioral, HR, or SCR level. For clarity, corresponding descriptive and inferential statistics are therefore not presented here.

(Manuscript received August 10, 2005; revision accepted for publication March 24, 2006.) 